\title{
Modeling a high velocity LMC: The formation of the Magellanic Stream
}

\author{
Chiara Mastropietro ${ }^{1,2}$ \\ ${ }^{1}$ LERMA, Observatoire de Paris, UPMC, CNRS, 61, A. de l'Observatoire, \\ 75014, Paris, France, \\ email: chiara.mastropietro@obspm.fr \\ ${ }^{2}$ Universitäts Sternwarte München, Scheinerstr.1, D-81679 München, Germany
}

\begin{abstract}
I use high resolution N-body/SPH simulations to model the new proper motion of the Large Magellanic Cloud (LMC) within the Milky Way (MW) halo and investigate the effects of gravitational and hydrodynamical forces on the formation of the Magellanic Stream (MS). Both the LMC and the MW are fully self consistent galaxy models embedded in extended cuspy $\Lambda \mathrm{CDM}$ dark matter halos. I find that ram-pressure from a low density ionized halo is sufficient to remove a large amount of gas from the LMC's disk forming a trailing Stream that extends more than 120 degrees from the Cloud. Tidal forces elongate the satellite's disk but do not affect its vertical structure. No stars become unbound showing that tidal stripping is almost effectless.
\end{abstract}

Keywords. methods: n-body simulations, Galaxy: halo, galaxies: individual (LMC), galaxies: interactions, galaxies: kinematics and dynamics, Magellanic Clouds

\section{Introduction}

Recent HST proper motion measurements of the Magellanic Clouds by Kallivayalil et al. (2006), Kallivayalil, van de Marel \& Alcock (2006) and Piatek et al. (2008) indicate that they are presently moving at velocities substantially higher (almost $100 \mathrm{~km} \mathrm{~s}^{-1}$ ) than those provided by previous observational studies (van der Marel et al. 2002, Kroupa \& Bastian 1997). Such high velocities $\left(v=378 \mathrm{~km} \mathrm{~s}^{-1}\right.$ and $v=302 \mathrm{~km} \mathrm{~s}^{-1}$ for the LMC and SMC, respectively) are close to the escape velocity of the Milky Way and consistent with the hypothesis of a first passage about the Galaxy (Besla et al. 2007). A single perigalactic passage has serious implications for the origin of the Magellanic Stream. It definitely rules out the tidal stripping hypothesis (Růžička, Theis \& Palouš 2008) since in this scenario the loss of mass is primarily induced by tidal shocks suffered by satellites at the pericenters (Mayer et al. 2006) and the Stream would not have time to form before the present time. Indeed kinematical data suggest that the Clouds are now just after a perigalactic passage. On the other hand, ram-pressure scales as $v^{2}$, where $v$ is the relative velocity between satellites and the ambient medium. The high velocities of the Clouds could therefore compensate the effect of the reduced interaction time with the hot halo of the MW and hydrodynamical forces would play a determinant role in forming the Stream.

In Mastropietro et al. (2005, hereafter M05) we have performed high resolution Nbody/SPH simulations to study the hydrodynamical and gravitational interaction between the LMC and the MW using orbital constraints by van der Marel et al. (2002) and a present time satellite velocity of $250 \mathrm{~km} \mathrm{~s}^{-1}$. We found that, after two perigalactic passages, the combined effect of tidal forces and ram-pressure stripping can account for the majority of the LMC's internal features and for the formation of the MS. More in detail, ram-pressure stripping of cold gas from the LMC's disk produces a Stream with 
morphology and kinematics similar to the observed ones, while tidal stripping has longer time-scales and is not efficient in forming stellar debris, consistently with the lack of stars observed in the Stream. Nevertheless, at each pericentric passage the LMC suffers tidal heating which perturbs the overall structure of the satellite reducing the gravitational restoring force and therefore indirectly contributing to the loss of gas.

The main objection to this model, in light of the new proper motion measurements of the LMC, is that the time spent by the LMC within the hot halo of the MW would be to short to cover the full extension of the Stream (more than 100 degrees) by ram-pressure mechanisms (Besla et al. 2007). Moreover, hydrodynamical forces would affect a galaxy only weakly perturbed by the gravitational interaction, and stripping would result more difficult.

In this work I present the results of N-body/SPH simulations where the interaction between the MW and the LMC is modeled according to the new proper motion measurements of Kallivayalil et al. (2006a).

\section{Galaxy models}

The initial condition of the simulations are constructed using the technique described by Hernquist (1993). Both the MW and the LMC are multi-component galaxy models with a stellar and gaseous disk embedded in a spherical dark matter halo. The density profile of the NFW halo is adiabatically contracted due to baryonic cooling. Stars and cold gas in the disks follow the same exponential surface density profile. We also explore the eventuality of an extended LMC gaseous disk. In this model the gaseous disk is characterized by an additional constant density layer which extends up to eight times the scale length of the exponential disk. The MW model comprises also a small stellar bulge and an extended low density $\left(n=2 \times 10^{-5} \mathrm{~cm}^{-3}\right.$ within $150 \mathrm{kpc}$ from the Galactic center and $n=8.5 \times 10^{-5} \mathrm{~cm}^{-3}$ at $\left.50 \mathrm{kpc}\right)$ hot $\left(T=10^{6} \mathrm{~K}\right)$ halo in hydrostatic equilibrium inside the Galactic potential (M05). The MW model, with virial mass $10^{12} \mathrm{M}_{\odot}$ and concentration $c=11$, is similar to model A1 of Klypin et al. (2002) while the structural parameters of the LMC are chosen in such a way that the resulting rotation curve resemble that of a typical bulge-less late-type disk galaxy. In detail, the satellite has virial mass $2.6 \times 10^{10} \mathrm{M}_{\odot}$, concentration $c=9.5$ and the same amount of mass in the stellar and gaseous disk component $\left(\sim 10^{9} \mathrm{M}_{\odot}\right)$. The Toomre's stability criterion is always satisfied and the parameter $Q$ set equal to 1.5 and 2.0 at the disk scale radius in the different LMC models.

\section{Simulations}

I performed adiabatic simulations using GASOLINE, a parallel tree-code with multistepping (Wadsleyet al. 2004). High resolution runs have $2.46 \times 10^{6}$ particles, of which $3.5 \times 10^{5}$ are used for the disks and $5 \times 10^{5}$ for the hot halo of the MW. The gravitational spline softening is set equal to $0.5 \mathrm{kpc}$ for the dark and gaseous halos, and to $0.1 \mathrm{kpc}$ for stars and gas in the disk and bulge components.

In my best model the LMC approaches the MW on an unbound orbit (Fig. 1) with initial Galactocentric distance of $400 \mathrm{kpc}$ and velocity $\sim 190 \mathrm{~km} \mathrm{~s}^{-1}$. After the perigalactic passage (at $\sim 40 \mathrm{kpc}$ ) the velocity decreases faster than for a ballistic orbit as a result of dynamical friction. The escape velocity at a given LMC position is indicated by a red curve in the right panel and calculated assuming a spherical unperturbed host potential. Due to the effects of dynamical friction, at late times the satellite lies on a nearly parabolic orbit. At the present time $(t \sim 1.78 \mathrm{Gyr}$, vertical lines in the plots $)$ it 

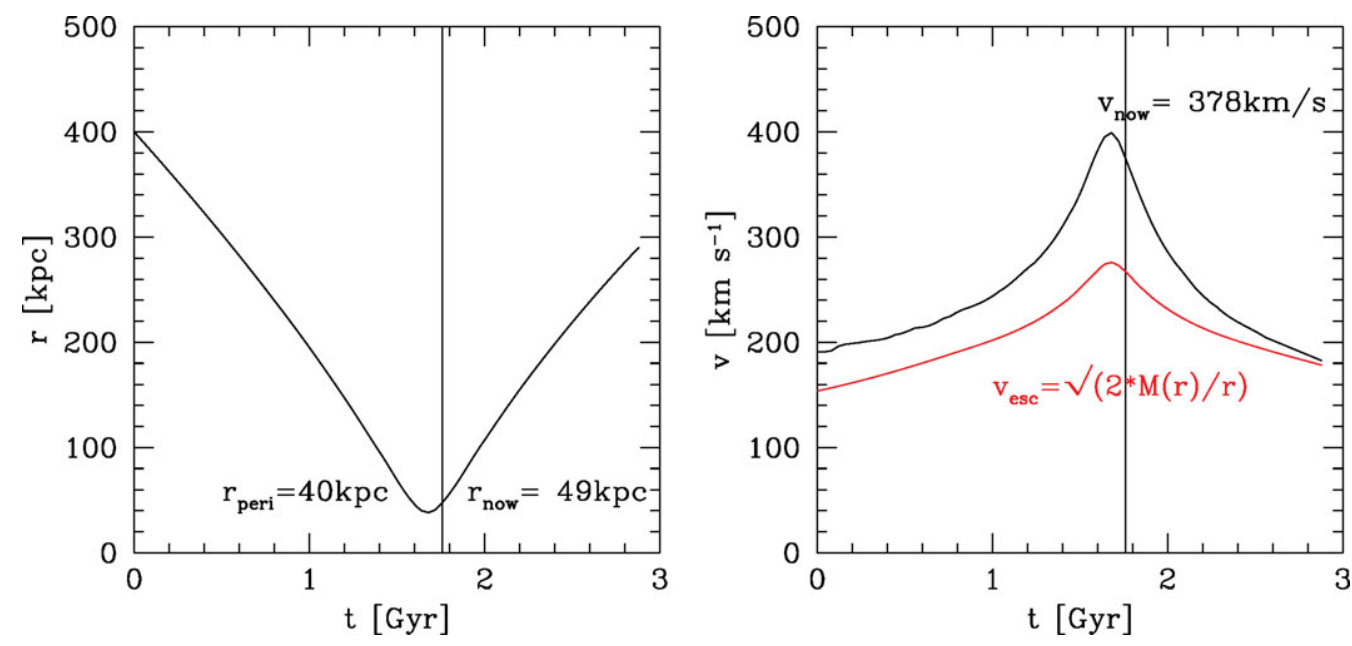

Figure 1. Orbit (left) and orbital velocity (right) of the LMC. Vertical lines represent the present time, just after the perigalacticon. Present values and values at the perigalacticon are indicated.

reaches a velocity of $378 \mathrm{~km} \mathrm{~s}^{-1}$ at $49 \mathrm{kpc}$ from the Galactic center, in good agreement with the new proper motion measurements.

In choosing the initial inclination of the LMC I made the approximation that it does not change during the interaction due to the effects of precession or nutation of the disk plane. At the beginning of the simulation the disk moves almost face-on through the external medium, and ram-pressure affecting the whole disk perpendicularly. In proximity to the perigalactic passage the velocity vector changes rapidly and the angle between the satellite's disk and the proper motion is close to zero. At the present time the simulated disk has an inclination of about 30 degrees with respect to the orbital motion (Kallivayalil et al. 2006a) and is indeed moving nearly edge-on through the external hot gas, with rampressure compressing its eastern side.

Fig. 2 illustrates the present time distribution of stars and gas originating from the LMC's disk. The stellar disk becomes elongated while tidal debris start forming after the perigalactic passage. But all stars stay bound to the satellite. Tidal heating does not perturb significantly the vertical structure of the disk that remains thin and does not create a warp, unlike what is observed in M05. Bar instability develops at the perigalacticon only in the case of $Q=1.5$.

Ram-pressure strips nearly $2 \times 10^{8} \mathrm{M}_{\odot}$ of gas from the LMC's disk forming a continuous Stream that lies in a thin plane perpendicular to the disk of the MW and extending up to $\sim 140$ degrees from the LMC (Fig. 3). The location of the Stream in the Southern Galactic hemisphere is comparable to the values of $b$ and $l$ provided by observations. Contrary to M05 there is no LMC's gas above the Galactic plane. In M05 the material lying in the Northern hemisphere is stripped from the satellite during the orbital period preceding the present one and the Stream forms a great polar circle around the Galaxy. The lack of gas at $b>0$ in Fig. 3 is not due to inefficient ram-pressure at early times, but to the fact that, in order to reproduce the current location and velocity of the LMC, the satellite enters the MW halo exactly at $b=0$.

The morphology of the Stream does not change significantly adopting an extended gaseous disk model (left panel of Fig. 3), except for the region at the head of the Stream, which appears broader (right). 

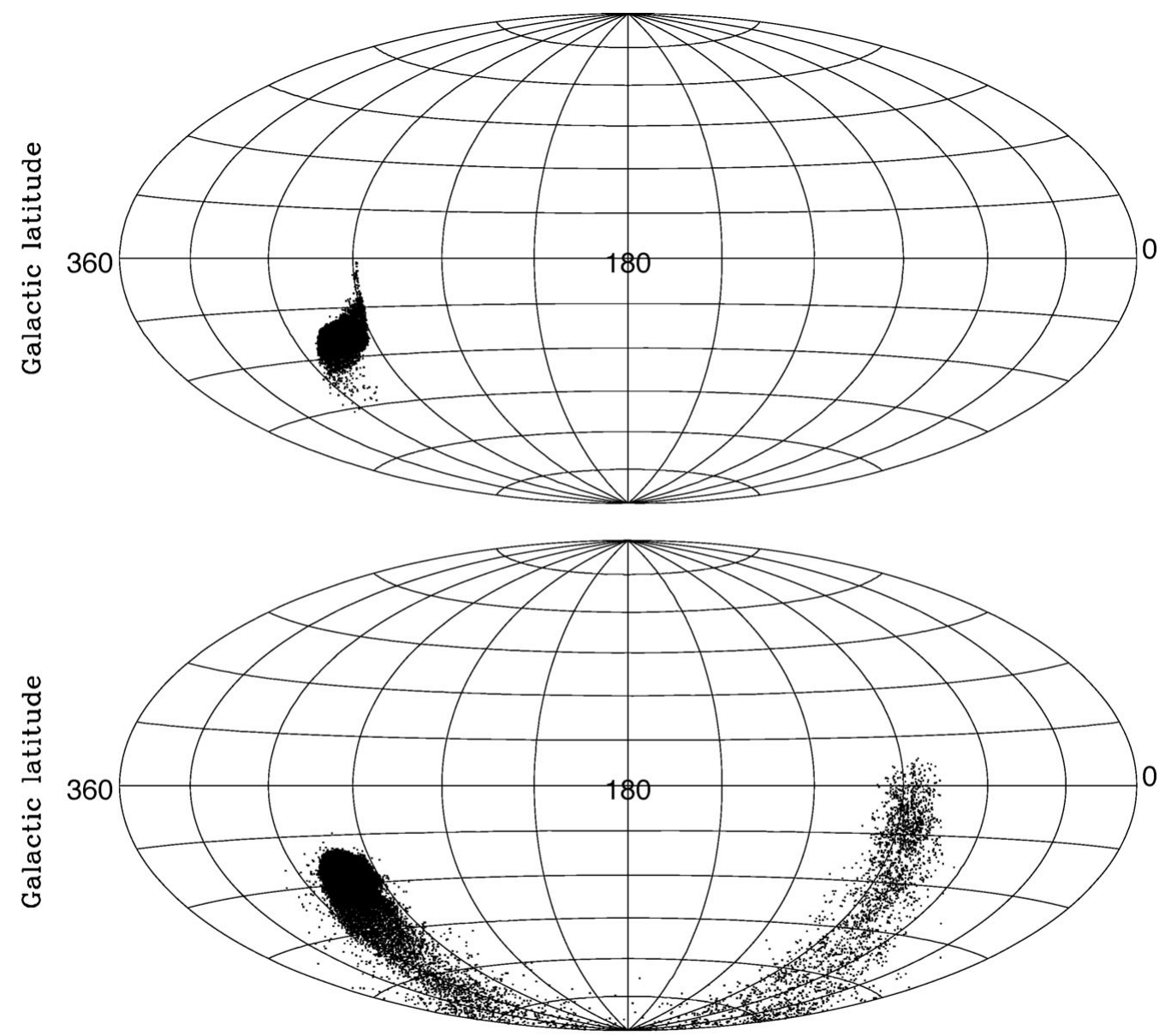

Galactic longitude

Figure 2. Present time distribution of stars (top) and gas (bottom) from the LMC disk in Galactic coordinates.

\section{Conclusions}

I carried out high resolution gravitational/hydrodynamical simulations of the interaction between the LMC and the MW using the orbital parameters suggested by the new HST proper motion measurements. I find that ram-pressure stripping exerted by a tenuous MW hot halo during a single perigalactic passage forms a Stream whose extension and location in the Sky are comparable to the observed ones. The stellar structure of the satellite is only marginally affected by tidal forces.

\section{Acknowledgements}

The numerical simulations were performed on the l SGI-Altix $3700 \mathrm{Bx} 2$ at the University Observatory in Munich. This work was partly supported by the DFG Sonderforschungsbereich 375 "Astro-Teilchenphysik". 

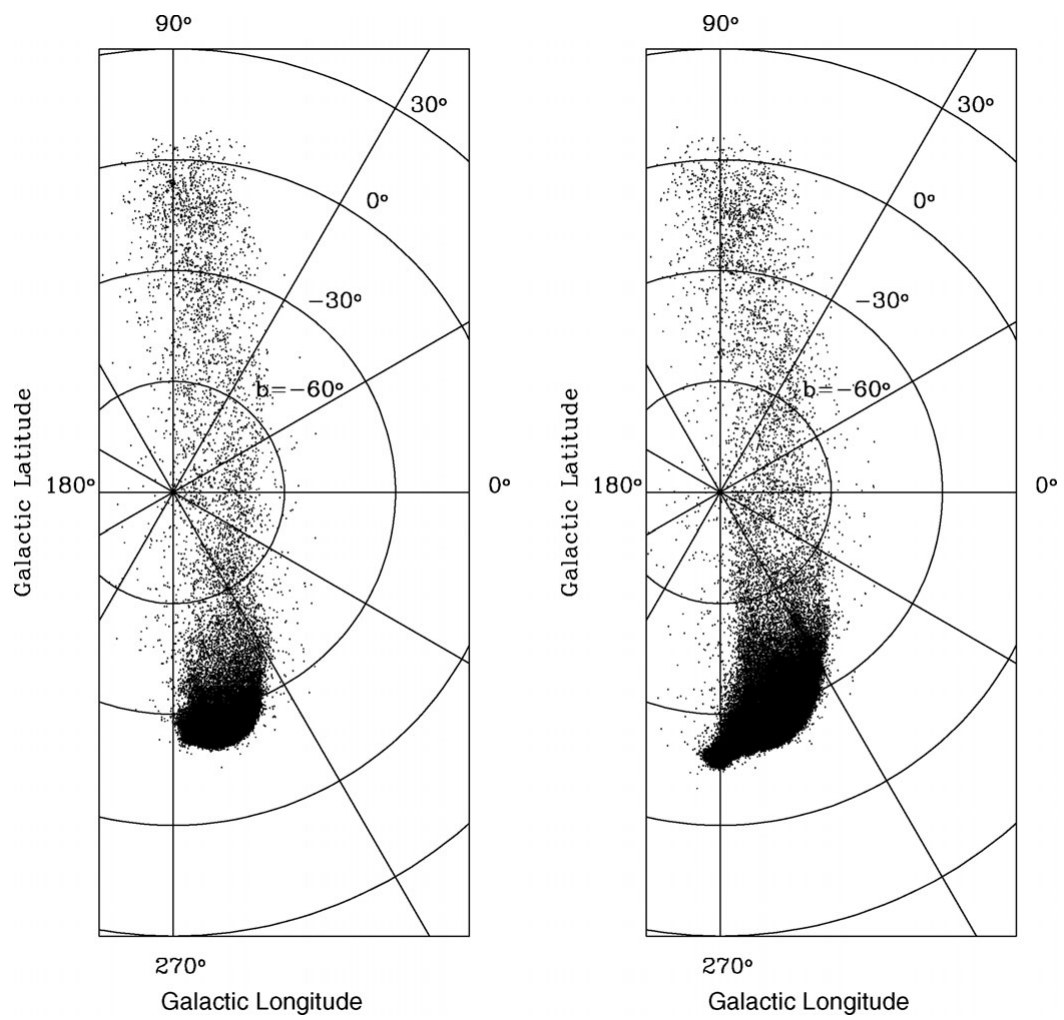

Figure 3. Polar projection of the simulated stream in Galactic coordinates. Both the pure exponential LMC model (left) and the model with extended disk (right) are shown.

\section{References}

Besla, G., Kallivayalil, N., Hernquist, L., Robertson, B., Cox, T. J., van der Marel, R. P., \& Alcock, C. 2007, ApJ, 668, 949

Hernquist, L. 1993, ApJS, 86, 389

Kallivayalil, N., van der Marel, R. P., Alcock, C., Axelrod, T., Cook, K. H., Drake, A. J., \& Geha, M. 2006a, ApJ, 638, 772

Kallivayalil, N., van der Marel, R. P., \& Alcock, C. 2006b, ApJ, 652, 1213

Klypin, A., Zhao, H., \& Somerville, R. S. 2002, ApJ, 573, 597

Kroupa, P. \& Bastian, U. 1997, New Astronomy, 2, 77

Mastropietro, C., Moore, B., Mayer, L., Wadsley, J., \& Stadel, J. 2005, MNRAS, 363, 509

Mayer, L., Mastropietro, C., Wadsley, J., Stadel, J., \& Moore, B. 2006, MNRAS, 369, 1021

Piatek, S., Pryor, C., \& Olszewski, E. W. 2008, AJ, 135, 1024

Růžička, A., Theis, C., \& Palouš, J. 2008, ApJ, in press, arXiv:0810.0968

van der Marel R. P., Alves D. R., Hardy E., \& Suntzeff N. B. 2002, AJ, 124, 2639

Wadsley, J. W., Stadel, J., \& Quinn, T. 2004, New Astronomy, 9, 137 